\title{
Charge transient and electrochemical measurements as a tool for characterization and degradation study of organic semiconductors - PMPSis and MEH-PPV
}

\author{
V. Nadáždy ${ }^{*}, 1$, K. Gmucová ${ }^{1}$, Š. Lányi $^{1}$, F. Schauer ${ }^{2, *}$, and I. Kuřitka ${ }^{2}$ \\ ${ }^{I}$ Institute of Physics, Slovak Academy of Sciences, Bratislava, Slovak Republic \\ ${ }^{2}$ Department of Electronics and measurement, Tomas Bata University, Zlin, Czech Republic \\ * Corresponding author.Tel: +421 220910761,E-mail:vojtech.nadazdy@savba.sk
}

\begin{abstract}
This contribution deals with application of several techniques based on charge transient contactless measurements (isothermal charge transient spectroscopy - IQTS), and by electrochemical methods of double step voltcoulometry and cyclic voltammetry, two complementary methods, which are potentially suitable for obtaining information about bulk relaxation and transport processes and the structure of electronic localized states and their basic parameters. Both methods were tested by two well known polymers, the first, Poly[methylphenylsilylene] and the second Poly(p-phenylene vinylene. The results were explained both in terms of bulk relaxation ant transport processes, trap parameters and the influence of UV degradation. The metastability in reconstruction of dangling bonds ensuing after the UV degradation due to the Si-Si $\sigma$ conjugated bond scission and its ability to reconstruct after the thermal anneal was again found in accord with the previous results.
\end{abstract}

Keywords: Organic semiconductors characterization, Electron structure, Transient charge method, Electrochemical method.

\section{Introduction}

The material research in organic semiconductors is moving towards the applications. When optimizing the properties of devices one has to start from the correct microphysical parameters of materials in question. The complicating factors in this direction is weak (molecular) coupling and the disordered structure, which both make the spectroscopic methods known from disordered inorganic semiconductors difficult and sometimes impossible to apply. So the need for new methods is acute and the new methods have been developed for organic material characterization.

It was shown that suitable techniques to electrical characterization of organic materials are space charge limited currents [1] and thermally stimulated currents [2]. As a favorable approach turns out capacitance or charge transient measurements which are processed by properly combined values of measured transients taken at specified time instants originally applied in deep-level transient spectroscopy of defect states in inorganic semiconductors. This transient processing allows to determine salient features of the transport states distribution [3] and charge traps induced by structural or chemical defects [4-6]. Electron transport based on hopping is important in polymers and localized molecular traps serve as redox sites. These facts authorize the application of solid-state electrochemical measurements. Some issues related to the principles and analytical aspects of electrochemical techniques were discussed in review by P.J. Kulesza and J.A. Cox [7].

Here we present two new methods with expressed advantages for the application in the field of molecular materials, isothermal charge transient spectroscopy and double state voltcoulometry. Both methods are based on the injection (extraction) contacts and the resulting signals in the time domain, which is analyzed for the capacitance and diffusion currents, respectively. Thus, we obtain information about capacitive (displacement) and 
diffusive (reduction-oxidation) phenomena that are quite complementary for the defects elucidation.

In the presented paper, we starting from the well known prototypical polymer Poly[methylphenylsilylene] (PMPSi), intend to test both the methods by comparison with previous results and then to apply it on quite important in the field of organic photovoltaics Poly(p-phenylene vinylene) (PPV).

\section{Methodology}

\subsection{Sample preparation}

Poly[methylphenylsilylene] (PMPSi) was purchased from Flurochem. PMPSi thin films were spin-coated from solution in toluene on the UV-ozone treated ITO substrates with a spin rate of $1500 \mathrm{rpm}$ for $30 \mathrm{~s}$ and dried in vacuum of $3 \times 10^{3} \mathrm{~Pa}$ at $60{ }^{\circ} \mathrm{C}$ for 4 hrs. Poly[2-methoxy-5(2'-ethyl-hexyloxy)-1,4-phenylene vinylene] (MEH-PPV) was supplied by Aldrich and solved in tetrahydrofuran. The MEH-PPV films were also spin-coated on the UV-ozone treated ITO substrates with a spin rate of $4800 \mathrm{rpm}$ for $30 \mathrm{~s}$ and dried in vacuum of $10^{4} \mathrm{~Pa}$ at $100{ }^{\circ} \mathrm{C}$ for 12 hrs. Exposition to UV irradiation + ozone of thin films under investigation were done with low-pressure mercury lamp with the total UV intensity at the sample surface of $2 \mathrm{~mW} / \mathrm{cm}^{2}$ and the ozone concentration was $150 \mathrm{mg} / \mathrm{m}^{3}$.

\subsection{Isothermal charge transient spectroscopy - IQTS}

The principle of the method IQTS is based on the time representation of the charge, induced in the external circuit by the driving pulse applied to the sample. This charge may be either due to the bulk relaxation processes, charge redistribution or release of charges from localized centres (traps). In this respect it is very similar to the method of post-transit extraction spectroscopy, where charges released from traps are time resolved collected and evaluated spectroscopically [1]. The IQTS spectrum is created by combining samples from charge transients at particular times using the formula $\Delta Q\left(t_{1}\right)=Q\left(t_{1}\right)-1.5 Q\left(\mathrm{t}_{2}=2 t_{1}\right)-0.5 Q\left(\mathrm{t}_{3}=\right.$ $4 t_{1}$ ) [8] (see Fig. 1). The rate window $\tau$ defined by this filter is $1 / t_{1}$ and it removes the linear component of the response, which is caused by the integration of direct current. The peak maximum corresponds to 0.174 of the total collected charge $Q_{0}$ responsible for the peak. Omitting the excitation pulse and the charge processing using the filter formula $I_{D C}=\left(Q\left(t_{2}\right)-\right.$ $\left.Q\left(t_{1}\right)\right) /\left(\mathrm{t}_{2}-\mathrm{t}_{1}\right)$ (see Fig.1) allows to evaluate direct current which flows through the sample. The measurement of current-voltage (I-V) characteristic is obtained by sweeping the bias voltage applied to the sample.

Electronic transport measurements were performed with charge transient processor (CTP) in local mode [9] when the usual evaporated top electrode is replaced by the tip of the scanning probe in form of a sharpened 80 - $\mu$ m-tungsten wire oriented perpendicularly to the surface. The charge to voltage converter has the resolution of hundreds of electrons and time resolved transients from $2 \mu$ s to hundreds of ms can be recorded. The duration of excitation pulses was set from 1 to $100 \mathrm{~ms}$, their amplitude from 2 to $5 \mathrm{~V}$, and the period from 147 to $547 \mathrm{~ms}$. To reduce the noise usually 50 transients were collected and averaged. The I-V characteristics were measured with the sweeping rate of $100 \mathrm{mV} / \mathrm{s}$. All IQTS and I-V measuremen were done at room temperature.

\subsection{Double step voltcoulometry - DSVCM}

The double step voltcoulometry (DSVCM) is a complementary method for studying the electrochemical processes in investigated material. While with the help of the electrical 
methods the concentration of the active species and their energies either for the capture or for the emission from a defect/deep trap is measured, electrochemical methods yield the potential at which either reduction or oxidation of species under consideration take place. Realizing that oxidation describes the loss of electrons, while the reduction describes the gain of electrons, the emission from electron traps can be regarded as an oxidation process, the capture of electrons in electron traps as a reduction process. On the contrary, for hole traps the emission of holes from hole traps can be regarded as a reduction process, the capture of holes in hole traps as an oxidation process. Thereby the electrochemical methods can serve as a complementary and powerful tool for the study of the defect states formation or removing in organic semiconductors. Recently, we have reported the electrochemical observation of the formation of hydrogen- and hydroxyl-related defects in pentacene thin films [10].

The electrochemical analyser used in this work, developed in our laboratory, is similar in function to CTP [11]. During the single potential scan both the ramp voltage (voltammetric wave) and the incremental charge (voltcoulometric signal) are obtained. The excitation pulse is switched on and the transient current flowing in external circuit is integrated and processed by the time-domain filter $\Delta Q\left(t_{1}\right)=Q\left(t_{1}\right)-2 Q\left(\mathrm{t}_{2}=5 t_{1}\right)-Q\left(\mathrm{t}_{3}=9 t_{1}\right)$. This filtering scheme analogous to filter for IQTS eliminates both the constant and linear components of the signal, i.e. suppression of both the steady-state and capacitive contributions of the transient current with respect to the diffusion current contribution. The amplitude of measured signal reflects not only the concentration of measured species, but also the kinetics of the measured charge transfer. A peak present only at the recorded voltcoulometric signal, which is not accompanied with a voltammetric wave, originates in the charging of an electrical double layer only.

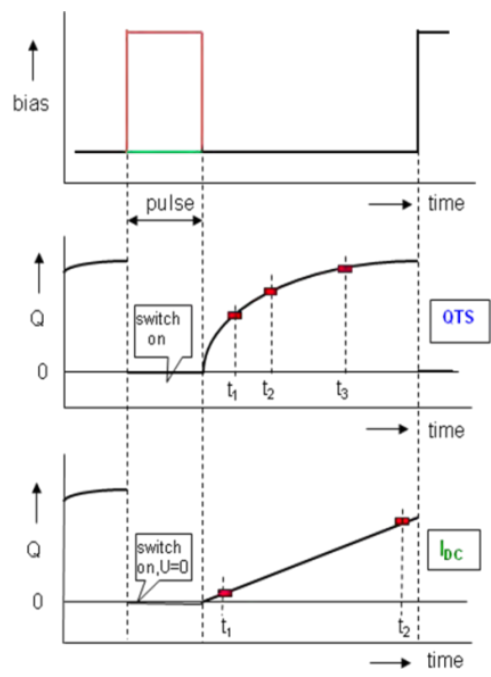

$$
\Delta Q=Q\left(t_{1}\right)+a Q\left(t_{2}\right)+b Q\left(t_{3}\right)
$$$$
I_{D C}=\left[\frac{Q\left(t_{2}\right)-Q\left(t_{1}\right)}{t_{2}-t_{1}}\right]
$$

Fig. 1. Timing chart of the applied voltage and measured charge transients used for isothermal charge transient spectroscopy (IQTS), direct current, $I_{D C}$, measurement, and double step voltcoulometry (DSVCM). The coefficients $a, b, t_{2}$, and $t_{3}$ are set as follows $a=-1.5, b=0.5, t_{2}=2 t_{1}, t_{3}=4 t_{1}$ for IQTS and $a=-2, b=1, t_{2}=5 t_{1}, t_{3}=9 t_{1}$ for DSVCM. 


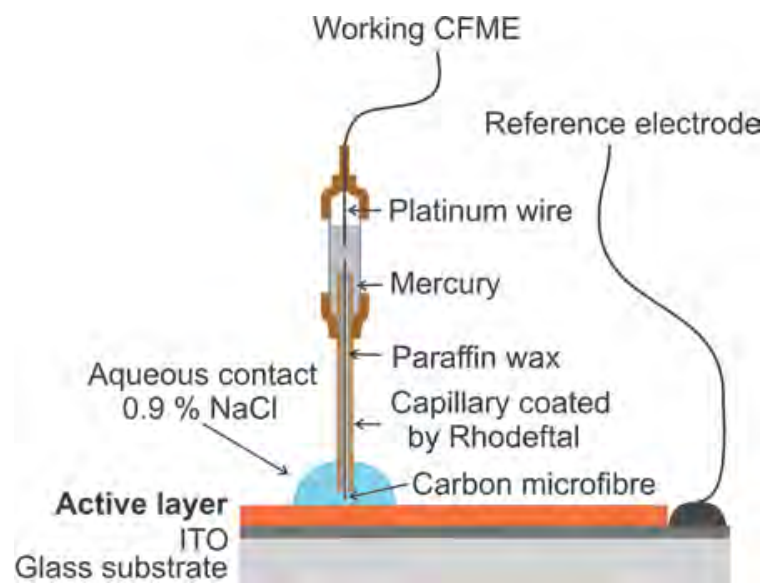

Fig. 2. Setup of electrochemical experiments.

The electrochemical experiments were carried out by the apparatus depicted in Fig. 2. The working carbon fiber microelectrode (CFME) was formed of a glass micropipette with up to eight carbon fibers (diameter of each fiber being approximately $7-8 \mu \mathrm{m}$ ). The pipette tip (diameter $100-150 \mu \mathrm{m}$ ) was filled with paraffin wax to prevent liquid from seeping inside around the carbon fibers. The exposed surfaces of the filaments were treated electrochemically before starting the experiments. First, a cathodic potential of - $0.8 \mathrm{~V}$ was applied for $40 \mathrm{~s}$, followed by a triangular waveform of 0 to $+3 \mathrm{~V}$ for $10 \mathrm{~s}$; finally, an anodic potential of $+1.5 \mathrm{~V}$ was applied for $10 \mathrm{~s}$. The reported results correspond to the measurements made for the sweeping rate of $33.3 \mathrm{mVs}^{-1}$, the potential step amplitude for the voltcoulometric period of measurement was set to $-0.1 \mathrm{~V}$ and to $0.1 \mathrm{~V}$ for the anodic and cathodic scans, respectively.

\section{Results}

The representative signals measured on the PMPSi samples are in Figs. 3, 4, 5 for the fresh sample and subsequently degraded by the UV in time steps 2, 5 and $10 \mathrm{~min}$. The remarkable increase in hole injection ability of the ITO contact was recorded (Fig.3a). The IQTS signal (Fig. 3b) measured on identical spots shows first neither the bulk relaxation processes or trapping states, on UV degradation gradual development of the hole trap states is observed, with the most expressed signal at $t_{1}=1.5 \mathrm{~ms}$ for degradation time $10 \mathrm{~min}$. To examine the time dependence of the emitted carriers from the traps, the influence of the extraction voltage $\left(U_{\mathrm{b}}\right)$, keeping the injecting charge constant (given by $U_{\mathrm{b}}-\Delta U$ ) were measured (see Fig. 4). The signal shifts to shorter times $t_{1}$ with increasing $U_{\mathrm{b}}$ as expected due to the shorter extraction. On the contrary when applying the injecting voltage on tungsten electrode, it results in virtually no filling of the trapping states and only small IQTS signal is detected.

The steady-state voltammetry and DSVCM signals on identical samples of PMPSi are in Fig. $5 \mathrm{a}$ for anodic scans (ramp voltage is going from $-1.5 \mathrm{~V}$ to $1.5 \mathrm{~V}$ ) and in Fig. $5 \mathrm{~b}$ for cathodic scans (from $1.5 \mathrm{~V}$ to $-1.5 \mathrm{~V}$ ). Steady-state voltammetry offers potential - current characteristics, where the presence of electrically active centre (defect) is detected by the presence of voltammetric wave, whose amplitude is proportional to the concentration of this centre. Therefore, the increasing current wave near the potential of $+0.8 \mathrm{~V}$ depicted in Fig. 5a corresponds to evolution of a defect in PMPSi, which is caused by the exposure of the PMPSi 
(a)

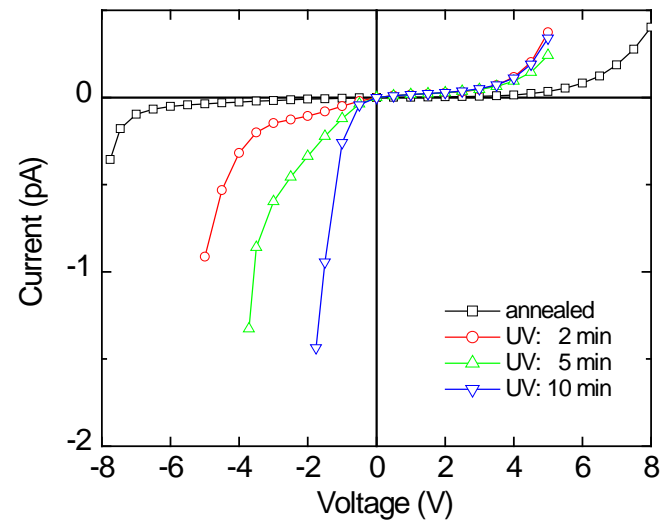

(b)

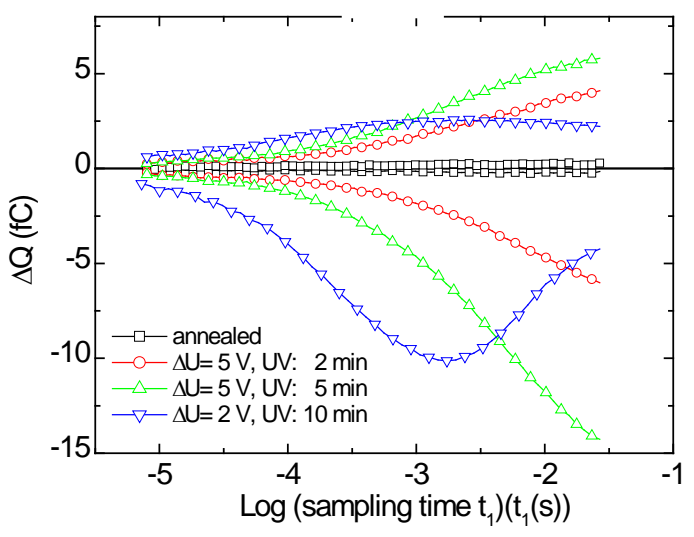

Fig. 3. Evolution of current - voltage characteristics (a) and IQTS spectra (b) of the structure ITO /PMPSi/ W probe with exposition time to UV irradiation. IQTS spectra in upper and lower parts correspond to excitation with positive and negative pulses. Bias voltage, $U_{b}=0$, the height of excitation pulses takes into account the electrical conductivity of the PMPSi film: $5 \mathrm{~V}$ for annealed, 2, and $5 \mathrm{~m}$ in for irradiated samples, $2 \mathrm{~V}$ for $10 \mathrm{~m}$ in irradiated sample. The period and duration of excitation pulses were set to $147 \mathrm{~ms}$ and $10 \mathrm{~ms}$, respectively.

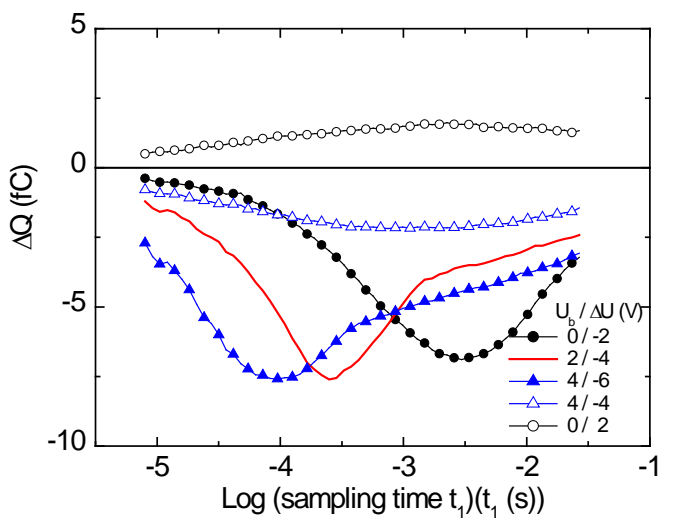

Fig. 4. Effect of bias voltage and the height of excitation pulses on the IQTS peak observed on ITO / $P M P S i$ / tungsten probe structure after 10 min of exposure to UV irradiation. The period and duration of excitation pulses were set to $147 \mathrm{~ms}$ and $10 \mathrm{~ms}$, respectively.

(a)

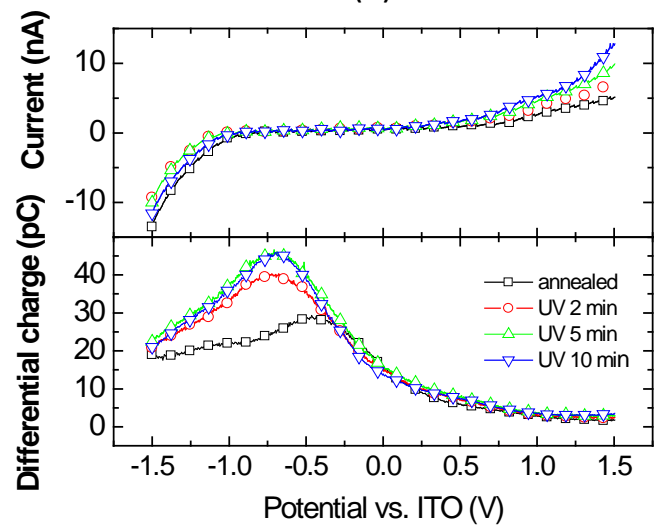

(b)

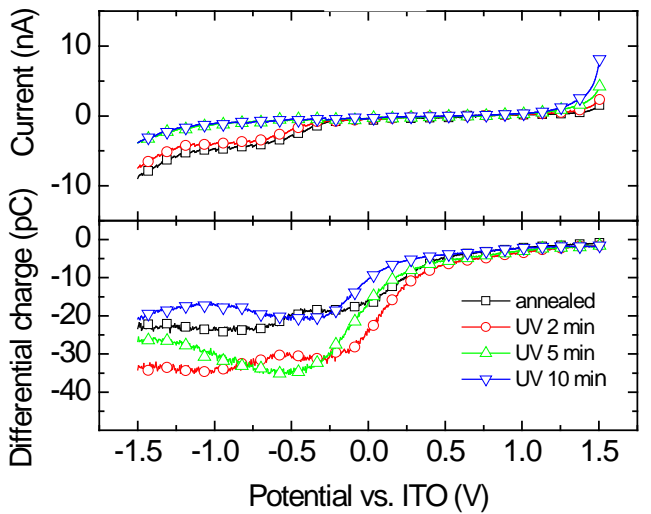

Fig. 5. Voltammetric (upper part) and voltcoulometric (lower part) signals obtained for PMPSi thin film during (a) anodic scans, i.e, for the potential swept from $-1.5 \mathrm{~V}$ to $1.5 \mathrm{~V}$ and (b) cathodic scan, i.e., for potential swept from $1.5 \mathrm{~V}$ to $-1.5 \mathrm{~V}$. Individual data sets have been obtained for various exposition times to UV irradiation. 
to UV irradiation. The position of voltcoulometric peak does not in general match the position of voltammetric wave on the I-V characteristic; the difference depends on the ratio of the apparent diffusion coefficients for the measured charge transfer of capture and emission of electrons or holes. Therefore, the tiny increase of voltcoulometric signal observed at potential $+0.5 \mathrm{~V}$ (Fig. 5a, lower part) can be related to the voltammetric wave at $+0.8 \mathrm{~V}$.

While the voltammetric wave (anodic scan) in Fig. 5a is caused by the capture of holes by trap or the emission of electrons from trap, voltammetric wave in Fig. 5b (cathodic scan) represents measurement of electron capture or hole emission from the trap. Thus, the discharge of the process at about $-0.5 \mathrm{~V}$ (observed on both the voltammetric and voltcoulometric signals) can be regarded as drop-out of the ability of the PMPSi to capture electrons or emit holes from a PMPSi matrix after 5-minute exposure to UV irradiation. It should be noted that I-V characteristic and IQTS spectrum of anneled state was recovered by annealing the sample in vacuum at $80^{\circ} \mathrm{C}$ for 1 hour.

(a)

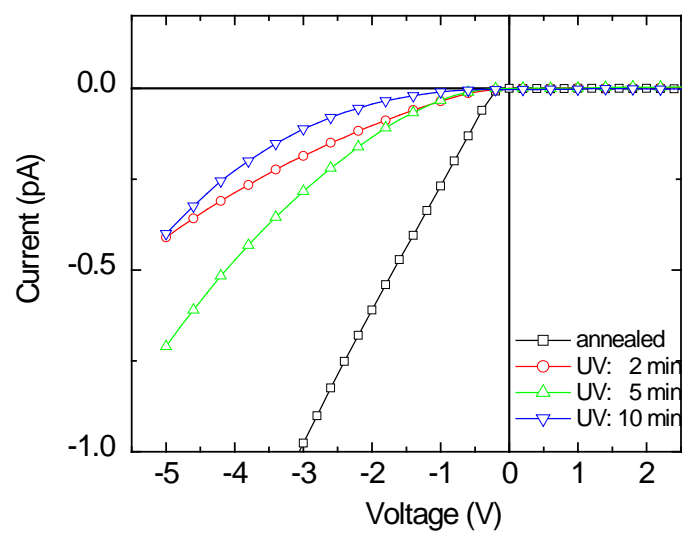

(b)

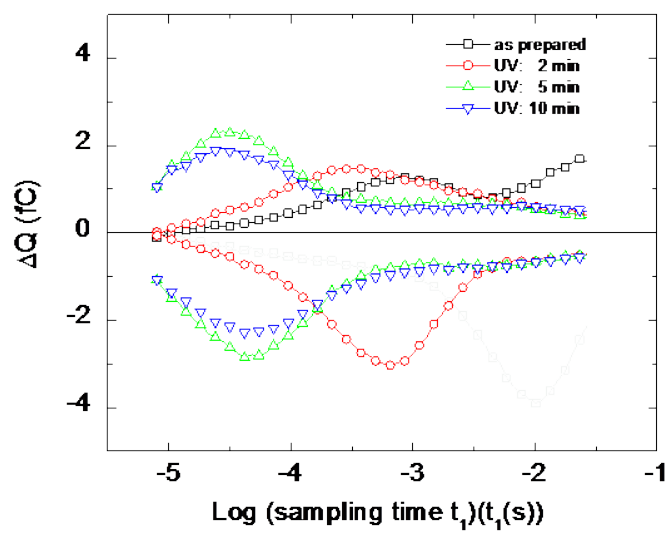

Fig. 6. Evolution of current - voltage characteristics (a) and IQTS spectra (b) of the structure ITO / $M E H-P P V /$ tungsten probe with exposition time to UV irradiation. IQTS spectra in upper and lower parts correspond to excitation with positive and negative pulses. Bias voltage, $U_{b}=0$, the height of excitation pulses was set to $5 \mathrm{~V}$ for annealed and exposed states. The period and duration of excitation pulses were set to $147 \mathrm{~ms}$ and $10 \mathrm{~ms}$, respectively.

(a)

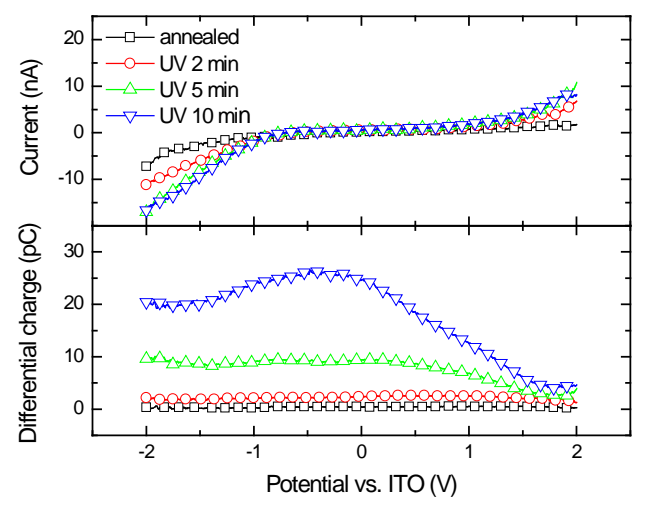

(b)

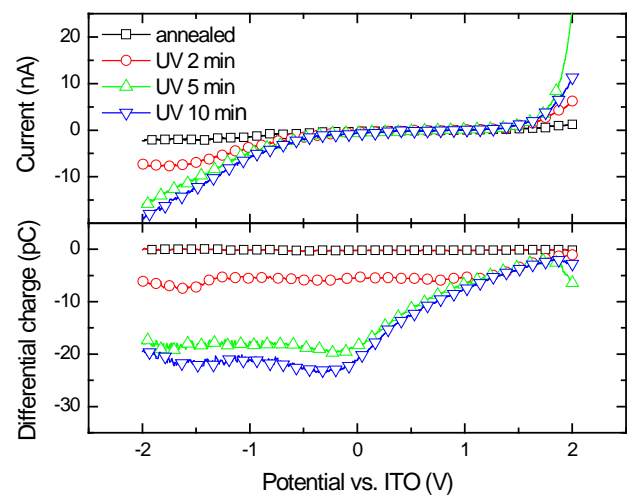

Fig. 7. Voltammetric (upper part) and voltcoulometric (lower part) signals obtained on MEH-PPV thin film during (a) anodic scan, i.e, for potential swept from $-1.5 \mathrm{~V}$ to $1.5 \mathrm{~V}$ and (b) cathodic scan, i.e, for potential swept from $1.5 \mathrm{~V}$ to $-1.5 \mathrm{~V}$. Individual data sets have been obtained for various exposition times to UV irradiation. 
Similarly, the signals in MEH PPV are visible in Fig. 6a (I-V) and Fig. 6b (IQTS). The behavior is quite different, as the injecting properties with UV irradiation decrease, contrary to PMPSi. The IQTS signals depict no trapping, but only bulk relaxation effects, testified by symmetrical signals for both polarities, independent on injection. Also, the DSVCM signals in Fig. 7a (anodic scan) and in Fig. 7b (cathodic scan) give evidence of the evolution of a charge transfer process caused by UV at about $-1.5 \mathrm{~V}$.

\section{Discussion and conclusions}

In Fig. 8 there are the results of post-transit spectroscopy on PMPSi achieved by large signal transient SCLC [1]. The extreme filling of the traps was achieved by laser injection. On UV degradation the $\mathrm{Si}-\mathrm{Si} \sigma$ bond breaking leads to the metastable states at about $0.55 \mathrm{eV}$, which are fully reconstructed on annealed [1]. The method is identical to IQTS with much less influence on the sample (UV laser used) due to its extreme sensitivity.

(a)

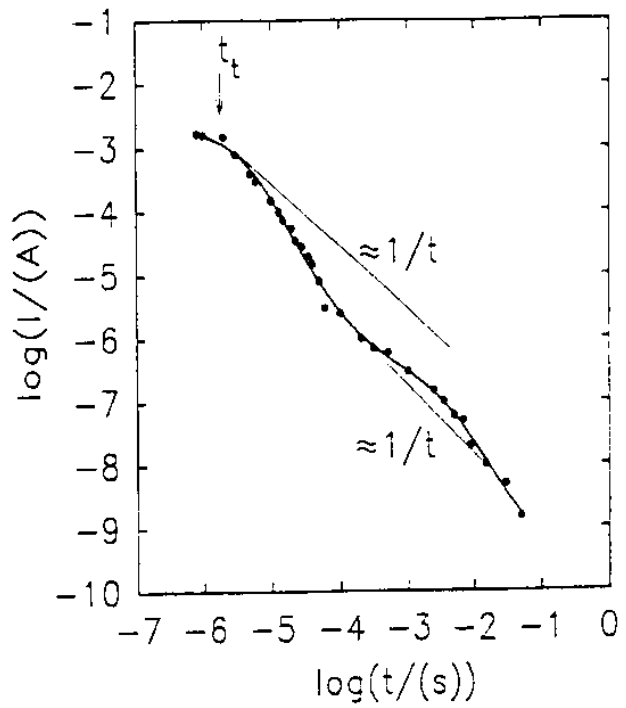

(b)

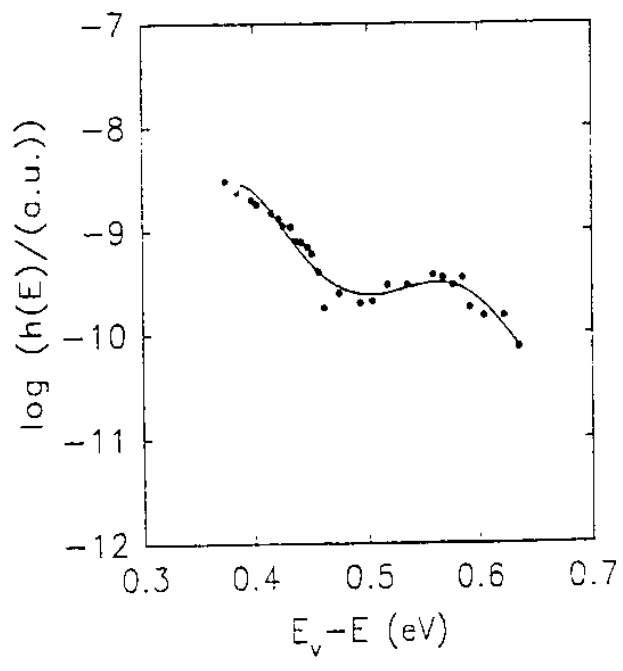

Fig. 8 The post-transit current in large signal SCLC on PMPSi (a), the corresponding DOS distribution (b).

UV irradiation causes bulk modification of both PMPSi and MEH-PPV films under investigation. It is remarkable that the same degradation treatment leads to opposite effect on I-V characteristics. The hole injection in the metal insulator metal (MIM) structure with PMPSi increases but that with MEH-PPV shows decrease of hole injection with degradation. The evolution of corresponding IQTS spectra (Fig. 3b and 6b), voltammetric, and voltcoulometric signals (Figs. 5 and 7) with time exposition to UV irradiation are rather complex. However, some common trends in their behavior can be inferred from the comparison with the evolution of I-V characteristics. Improvement of the hole injection for PMPSi leads to asymmetry of IQTS spectra for negative and positive polarity of excitation pulses. In voltamogram, decay of the current wave at negative potential of $-0.5 \mathrm{~V}$ is observed and the voltammetric wave at $+0.8 \mathrm{~V}$ is simultaneously developed. Deterioration of the injection for the structure with MEH-PPV is correlated with the development of symmetric 
IQTS peak located at about $50 \mu$ s and voltammetric wave at about -1.5V. There is a clear twostep increase of this wave and shift in the IQTS peak position.

Steady-state voltammetry offers I-V characteristics, where the presence of electrically active centre (defect) is detected by the presence of voltammetric wave, which amplitude is proportional to the concentration of this centre. Voltammetric wave definitely reveals the presence of redox centers, i.e. electrically active traps. It means that in PMPSi some deep centers (continuum, corresponding to tail of IQTS spectra) decays and a shallower one arises. The increasing current wave near the potential of $+0.8 \mathrm{~V}$ depicted in Fig. 3 corresponds to evolution of a defect center in PMPSi caused by the exposure of the PMPSi to UV irradiation, which mediates charge transfer through redox reaction. On the other hand, the process at about $-0.5 \mathrm{~V}$ (observed on both the voltammetric and voltcoulometric signals) can be regarded as drop-out of other redox centre in PMPSi matrix after 5-minute exposition to UV irradiation + ozone. This process is accompanied with more effective hole injection. In the case of MEH-PPV, voltammetric wave at $-1.5 \mathrm{~V}$ indicates a redox centre (trap). The IQTS peak position at $50 \mu$ s supposes relatively shallow trap. The determination of the activation will require IQTS measurements at several temperatures.

\section{Acknowledgements}

The work in Slovak Republic was supported by the ASFEU project Effective control of production and consumption of energy from renewable resources, Activity 4.2, ITMS code 26240220028, supported by the Research \& Development Operational Programme funded by the ERDF and by Slovak grant agency VEGA, projects 2/0093/10 and 2/0063/11.

\section{References}

[1] F. Schauer, Space-charge-limited photoconductivity in polymers, Czech. J. Phys. 49, 1999, p. 871.

[2] J. Steiger, R. Schmechel, H. von Seggern, Synthet. Metals 129, 2002, p. 1.

[3] A.J. Campbell, D.D.C. Bradley, E. Werner, W. Brutting, Organic Electr. 1, 2000, p. 21.

[4] Y.S. Yang, S.H. Kim, J. Lee, H.Y. Chu, L. Do, H. Lee, J. Oh, T. Zyung, Appl. Phys. Lett. 80, 2002, p. 1595.

[5] C. Renaud, T.P. Nguyen, J. Appl. Phys. 107, 2010, p. 124505.

[6] I. Thurzo, H. Mendez, D.R.T. Zahn, phys. stat. sol (a) 202, 2005, p. 1994.

[7] P.J. Kulesza, J.M. Cox, Electroanalysis 10, 1998, p. 73.

[8] I. Thurzo, K. Gmucová, Rev. Sci. Instrum. 65, 1994, p. 2244.

[9] Š. Lányi, V. Nádaždy, Ultramicroscopy 110, 2010, p. 685.

[10] K. Gmucová, M. Weis, M. Della Pirriera, J. Puigdollers, phys. stat. sol. (a) 206, 2009, p. 1404.

[11] I. Thurzo, K. Gmucová, J. Orlický, J. Pavlásek, Rev. Sci. Instrum. 70, 1999, p. 3723. 